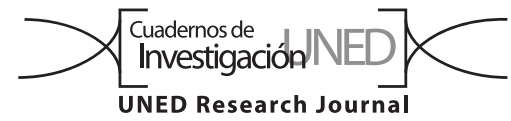

\title{
Mineralización de nitrógeno de rastrojo de vainica (Phaseolus vulgaris) en un suelo andisol tropical
}

\author{
Dayana Melissa Solórzano Quesada', Paola Brenes Rojas² \& Wagner Peña ${ }^{3}$ \\ 1. Universidad Estatal a Distancia, Bachillerato Ingeniería agronómica, San Pedro, San José, Costa Rica; \\ dayasq93@hotmail.com, (iD https://orcid.org/0000-0001-6771-2369 \\ 2. Universidad Estatal a Distancia de Costa Rica; Vicerrectoría de Investigación, San Pedro, San José, Costa Rica; \\ paolarj@@gmail.com, (D) https://orcid.org/0000-0003-0742-1273 \\ 3. Universidad Estatal a Distancia, Escuela de Ciencias Exactas y Naturales, Catedra de Gestión Sostenible del Suelo, San Pedro, San José, Costa \\ Rica; wpenaenator@gmail.com; (D) https://orcid.org/0000-0002-4678-0503
}

Recibido 27-V-2019 • Corregido 09-VIII-2019 • Aceptado 20-VIII-2019

DOI: https://doi.org/10.22458/urj.v11i3.2700

\begin{abstract}
Nitrogen mineralization from stubble degradation of green bean (Phaseolus vulgaris) in a tropical Andisol soil". Introduction: Mineralization is an essential process that improves the physical and chemical properties of soils through the recovery of biological activity and soil fertility, obtaining mainly mineral nitrogen used by plants. Objective: To determine the nitrogen contribution of green bean stubble (Phaseolus vulgaris) in an Andisol soil in San Ramón, Alajuela, Costa Rica. Methods: We did a chemical analysis ( $\mathrm{pH}$, acidity, $\mathrm{Ca}, \mathrm{Mg}, \mathrm{K}, \mathrm{P}, \mathrm{Cu}$, $\mathrm{Fe}, \mathrm{Zn}, \mathrm{Mn}$ ) on the soil before and after the experiment; and measured mineralization of the stubble incorporated in field plots after 10,20 and 30 days Results: The highest nitrogen contribution was at 30 days with $3,4 \%$ of the nitrogen contained in the stubbles, i.e. $79 \%$ of the total nitrogen. Conclusions: microbial activity, decomposition, nitrogen availability and use of carbon are best after 30 days.
\end{abstract}

Key words: Mineralization, nitrogen, Phaseoulus vulgaris, carbon, degradation, fertility, green beans.
RESUMEN: Introducción: La mineralización es un proceso esencial que mejora las propiedades físicas y químicas de los suelos mediante la recuperación de la actividad biológica y la fertilidad de los suelos, obteniendo principalmente nitrógeno mineral utilizado por las plantas. Objetivo: Determinar el aporte de nitrógeno de los rastrojos de vainica (Phaseolus vulgaris) durante un periodo de 30 días de mineralización en suelo andisol, en la zona de San Ramón, Alajuela, Costa Rica. Métodos: Realizamos un análisis químico ( $\mathrm{pH}$, acidez, $\mathrm{Ca}, \mathrm{Mg}, \mathrm{K}, \mathrm{P}, \mathrm{Cu}, \mathrm{Fe}, \mathrm{Zn}, \mathrm{Mn}$ ) en el suelo antes y después del experimento. A los 10, 20 y 30 días de la degradación del rastrojo se analizaron muestras. Resultados: El mayor aporte de nitrógeno fue a los 30 días con $3,4 \%$ del nitrógeno que contenían los rastrojos, representado un 79,07\% del total de nitrógeno. Conclusiones: A los 30 días de mineralización se presenta la mayor actividad microbiana, mejor descomposición de los rastrojos, disponibilidad de nitrógeno y el mejor aprovechamiento de carbono.

Palabras clave: Mineralización, nitrógeno, Phaseoulus Vulgaris, carbono, degradación, fertilidad, vainica.
La materia orgánica aporta a los suelos nitrógeno mineral utilizable por las plantas, obteniendo entre el 50 al $80 \%$ de las necesidades de nitrógeno para los cultivos, el cual se libera o retiene para mantener la productividad tanto a corto como largo plazo (Martínez, Galantini, López, \& Duval, 2014), esto se da gracias al proceso de mineralización el cual favorece la fertilidad de los suelos y a su vez la actividad biológica de los mismos (Jaramillo, 2016).

Mediante la taza de mineralización de los rastrojos en el suelo se logra conocer la máxima cantidad de nitrógeno que puede ser liberada en un periodo de tiempo, tomando en cuenta la mineralización acumulada. Cabe destacar que esta liberación se encuentra influenciada por diversas condiciones, como la composición del material, factores ambientales y características del suelo (Figueroa, Álvarez, Forero, Salamanca, \& Pinzón, 2012).

Además, el proceso de mineralización es de gran importancia para los ecosistemas agrícolas debido a la disminución de materia orgánica en los suelos por la intensificación de la agricultura (Martínez et al., 2014), por tal razón es necesario implementar medidas agroecológicas.

Una de las opciones es el uso de rastrojos de leguminosas, ya que pueden aportar entre 2,5 y $4 \mathrm{~kg} / \mathrm{m}^{2}$ de material vegetal y mediante su mineralización pueden 
proporcionar hasta $100 \mathrm{~kg}$ de N/ha por ciclo, ayudando a su vez a mejorar la calidad de los suelos (Guanche, 2012).

La planta de vainica (Phaseolus vulgaris), es una leguminosa que puede ser utilizada como abono verde al finalizar su ciclo como cultivo principal, esta especie es una forma mejorada del frijol y es conocida con diferentes nombres según sea el país como poroto verde, ejote, habichuela y vainica en Costa Rica (MAG, 1999). Dicha planta pertenece al reino Plantae, división Tracheophyta, clase Angiospermopsida, orden Fabales, familia Fabaceae, género: Phaseolus y especie Phaseolus vulgaris (Vargas, 2011).

La vainica es considerada una planta anual, caracterizada por un ciclo de florecimiento, producción de semillas y muerte. Las épocas de siembra son: mayo, octubre-noviembre, diciembre y enero, este cultivo necesita entre 300 a $400 \mathrm{~mm}$ de lluvia, se recomienda que los suelos sean profundos, fértiles, preferiblemente de origen volcánico con no menos de $1,5 \%$ de materia orgánica en la capa arable y de textura liviana como los francos, francos limosos y franco arcilloso, el pH óptimo está comprendido entre 6,5 y 7,5 aunque es tolerante a unidades de 4,5 y 8,2 (MAG, 1999).

El cultivo de vainica es de importancia en la dieta de los consumidores y accesible para los productores para ser aprovechada como abono verde (Delgado, Salvador, Morales, López, \& Rocandio, 2015). Según el Censo Agropecuario realizado en el 2014 por el Instituto Nacional de Estadística y Censos (INEC, 2014) en Costa Rica hubo un total de fincas con cultivo de vainica de 1 123, además según el Programa Integral de Mercadeo Agropecuario (PIMA, 2016) para el 2015 hubo un consumo de vainica de $2,9 \%$ del total de hortalizas más consumidas en los hogares costarricenses, presentando a su vez un consumo per cápita para ese mismo año de $246,55 \mathrm{~kg}$ por semana.

Por lo tanto, la vainica al ser una leguminosa común en las fincas y presentando concentraciones de nitrógeno en los órganos de hasta 54\% (Escalante \& Rodríguez, 2015) y aportes de nitrógeno de hasta $65 \mathrm{~kg} / \mathrm{ha}$ (Piccinetti, García, \& Perticari, 2015), puede ser utilizada como abono verde para el mejoramiento de suelos.

Es por ello que para obtener el mayor provecho en la utilización de los rastrojos de vainica (Phaseolus vulgaris) es importante tener en cuenta su proceso de mineralización, así como su aporte de nitrógeno, logrando con ello dar el manejo adecuado a los residuos de cosecha y a su vez tomar en cuenta su aporte de nutrientes en los planes de fertilización para evitar la contaminación y el desperdicio por exceso de nitrógeno no aprovechado (Jaramillo, 2016).

\section{MATERIALES Y MÉTODOS}

La investigación se llevó acabo en San Ramón, Alajuela $\left(10^{\circ} 06^{\prime} 33.0^{\prime \prime} \mathrm{N}, 84^{\circ} 26^{\prime} 32.1^{\prime \prime} \mathrm{O}\right)$ en un suelo andisol. El diseño utilizado fue de tipo experimental con enfoque cuantitativo, teniendo como variable independiente el rastrojo de vainica, esto con el fin de observar sus efectos sobre la variable dependiente, identificada como el aporte total de nitrógeno.

Se presentó una precipitación promedio de $8,2 \mathrm{~mm}$ y temperaturas promedio de $16^{\circ} \mathrm{C}$ esto según los datos proporcionados por el Instituto Meteorológico Nacional, la humedad del suelo fue de $78,05 \%$ al inicio y $73,7 \%$ al finalizar la investigación.

La investigación se centró en determinar el aporte de nitrógeno de los rastrojos de vainica (Phaseolus vulgaris) y las propiedades químicas de un suelo andisol. El rastrojo de vainica (Phaseolus vulgaris) compuesto por hojas se recolectó posterior a la cosecha, y se colocó en nueve bolsas de nylon de $20 \mathrm{~cm} \times 20 \mathrm{~cm}$, con $25 \mathrm{~g}$ de material cada una, seguidamente se incorporaron en nueve macetas con suelo respectivamente. También se incorporó el rastrojo completo en una parcela de campo de $1 \mathrm{~m}$ por $2,5 \mathrm{~m}$, esto mediante la extracción de la planta completa y su incorporación en los primeros $25 \mathrm{~cm}$ de la superficie del suelo. Al cabo de 10, 20 y 30 días se recolectaron las muestras foliares de las macetas utilizando un balde de plástico limpio, para transportar la muestra (bolsas de nylon con rastrojo), siendo secada cuidadosamente con papel toalla, y empacada e identificada en bolsas de plástico limpias para ser enviada al laboratorio donde se analizó a los rastrojos: nitrógeno total foliar, carbono total foliar, diferencia de peso en gramos, relación carbono/ nitrógeno. En el tratamiento 2 (parcela de campo) no se logró determinar los cambios de aprovechamiento foliar por error instrumental, ambiental y de tiempo.

El suelo se muestreó antes y después del experimento realizando un análisis químico completo $(\mathrm{pH}$, acidez, $\mathrm{Ca}, \mathrm{Mg}, \mathrm{K}, \mathrm{P}, \mathrm{Cu}, \mathrm{Fe}, \mathrm{Zn}, \mathrm{Mn}$ ), con el fin de comparar las propiedades de éste al inicio y al final de la incorporación de los rastrojos. En la parcela de campo se utilizó un muestreo sistemático tomando muestras del suelo en zigzag a $30 \mathrm{~cm}$ de profundidad, obteniendo una muestra compuesta a la cual se le aplico la técnica del cuarteo (división del suelo homogenizado en cuatro partes iguales y eliminando de los cuartos opuestos) para obtener una muestra representativa de $500 \mathrm{~g}$, esta se empacó en una bolsa de plástico limpia y se identificó para ser enviada al laboratorio; en el caso del tratamiento en macetas se recolectó el suelo de las mismas, se realiza la técnica de cuarteo obteniendo una cantidad de $500 \mathrm{~g}$ siendo empacada, identificada y enviada al laboratorio. 
En la investigación se utilizó un diseño de bloques completos al azar, compuesto por los dos tratamientos y sus tres repeticiones; la unidad experimental comprendió las nueve mesetas y la parcela de campo.

Todas las muestras fueron analizadas en el laboratorio del INTA (Instituto Nacional de Innovación y Transferencia de Tecnología Agropecuaria), también se realizó el análisis de los datos mediante el método estadístico "Análisis de Varianza" a través del programa INFOSTAT con un 5\% de error permitido.

Ética, conflicto de intereses y declaración de financiamiento: Los autores declaran haber cumplido con todos los requisitos éticos y legales pertinentes, tanto durante el estudio como en el manuscrito; que no hay conflictos de interés de ningún tipo, y que todas las fuentes financieras se detallan plena y claramente en la sección de agradecimientos. Asimismo, están de acuerdo con la versión editada final del documento. El respectivo documento legal firmado se encuentra en los archivos de la revista.

\section{RESULTADOS}

Características químicas del suelo al inicio y al final del experimento: En el Cuadro 1 se observan las características químicas del suelo posterior a la mineralización del rastrojo de vainica, en donde se enfatiza el aumento del $\mathrm{pH}$, presentando inicialmente 5,3, en $\mathrm{T} 1$ (=tratamientos en macetas) 5,5 y en T2 (=Tratamiento en parcela de campo) 5,6, así como la acidez presentando inicialmente 0,1 , en $\mathrm{T} 1$ 0,3 y en $\mathrm{T} 2$ 0,2. A su vez en el complejo de cambio se da un aumento en el potasio con $0,60 \mathrm{cmol}(+) / \mathrm{L}$ inicialmente, en $\mathrm{T} 10,77 \mathrm{cmol}(+) / \mathrm{L}$ y en $\mathrm{T} 20,73 \mathrm{cmol}(+) / \mathrm{L}$, con respecto al calcio se presenta un valor inicial de $6,5 \mathrm{cmol}(+) / \mathrm{L}$ que disminuye en $\mathrm{T} 1$ a $3,8 \mathrm{cmol}(+) / \mathrm{L}$ y aumenta en $\mathrm{T} 2$ a $8,2 \mathrm{cmol}(+) / \mathrm{L}$; del mismo modo el magnesio presentando inicialmente $1,5 \mathrm{cmol}(+) / \mathrm{L}$, que disminuye en $\mathrm{T} 1$ a $1,0 \mathrm{cmol}(+) / \mathrm{L}$ y aumenta en $\mathrm{T} 2$ a $2,2 \mathrm{cmol}(+) / \mathrm{L}$.

Por su parte también se obtiene un aumento en el fósforo inicialmente con un valor de $13 \mathrm{cmol}(+) / \mathrm{L}$ y aumentando en $\mathrm{T} 1$ a $36 \mathrm{cmol}(+) / \mathrm{L}$ y en $\mathrm{T} 2$ a $30 \mathrm{cmol}(+) / \mathrm{L}$, de forma contraria que el zinc ya que disminuyó teniendo $8,0 \mathrm{mg} / \mathrm{L}$ inicialmente, en $\mathrm{T} 17,0 \mathrm{mg} / \mathrm{L}$ y en $\mathrm{T} 26,0 \mathrm{mg} / \mathrm{L}$; por su parte el hierro, cobre y manganeso aumentan en T1 y disminuyen en T2, presentando inicialmente en hierro $192 \mathrm{mg} / \mathrm{L}$, en T1 $239 \mathrm{mg} / \mathrm{L}$ y en T2 $121 \mathrm{mg} / \mathrm{L}$, en cobre inicialmente $28 \mathrm{mg} / \mathrm{L}$, en T1 $30 \mathrm{mg} / \mathrm{L}$ y en T2 $14 \mathrm{mg} / \mathrm{L}$, del mismo modo en manganeso inicialmente con $52 \mathrm{mg} / \mathrm{L}$, en T 100mg/L en T2 47mg/L.

En el Cuadro 2 se presentan las relaciones catiónicas del complejo de cambio del suelo en el cual se llevó a cabo el análisis posterior a la mineralización de los rastrojos de vainica (Phaseolus vulgaris), en este se puede observar que la relación $\mathrm{Ca}+\mathrm{Mg}+\mathrm{K}$ es de $8,6 \mathrm{cmol}(+) / \mathrm{L}$ inicialmente, en $\mathrm{T} 1 \mathrm{con} 6,73 \mathrm{cmol}(+) / \mathrm{L}$, y en $\mathrm{T} 211,13 \mathrm{cmol}(+) / \mathrm{L}$; la relación $\mathrm{Ca} / \mathrm{Mg}$ en $\mathrm{T} 1$ presenta $3,8 \mathrm{cmol}(+) / \mathrm{L}$ y en $\mathrm{T} 23,72 \mathrm{cmol}(+) / \mathrm{L}$, seguidamente se presenta la relación $\mathrm{Ca} / \mathrm{K}$ con $4,93 \mathrm{cmol}(+) / \mathrm{L},(\mathrm{Ca}+\mathrm{Mg}) / \mathrm{K}$ con $6,23 \mathrm{cmol}(+) / \mathrm{L}$ y $\mathrm{Mg} / \mathrm{K}$ con $1,29 \mathrm{cmol}(+) / \mathrm{L}$, y en $\mathrm{T} 2$ mostrando en $\mathrm{Ca} / \mathrm{K} 11,23 \mathrm{cmol}(+) / \mathrm{L},(\mathrm{Ca}+\mathrm{Mg}) / \mathrm{K}$ con $14,24 \mathrm{cmol}(+) / \mathrm{L}$ y $\mathrm{Mg} / \mathrm{K}$ con $3,01 \mathrm{cmol}(+) / \mathrm{L}$, finalmente se presenta una saturación de acidez inicialmente de 1,1 , en $\mathrm{T} 1$ de 5,1 y en T2 de 1,76.

El contenido de carbono y nitrógeno en el suelo se reflejan en el Cuadro 3 en donde el porcentaje de carbono

CUADRO 1

Características químicas del suelo durante la mineralización de los rastrojos de vainica (Phaseolus vulgaris)

\begin{tabular}{cccccc} 
Unidad & Variable & Inicial 0 días & T1 20 días & T2 30 días & Ref. \\
& $\mathrm{pH}\left(\mathrm{H}_{2} \mathrm{O}\right)$ & 5,3 & 5,5 & 5,6 & $5,6-6,5$ \\
& $\mathrm{Acidez}$ & 0,1 & 0,3 & 0,2 & $0,5-1,5$ \\
$\mathrm{H} \mathrm{cmol}(+) / \mathrm{L}$ & $\mathrm{K}$ & 0,60 & 0,77 & 0,73 & $0,2-0,6$ \\
& $\mathrm{Ca}$ & 6,5 & 3,8 & 8,2 & $4-20$ \\
& $\mathrm{Mg}$ & 1,5 & 1,0 & 2,2 & $1-5$ \\
$\mathrm{mg} / \mathrm{L}$ & $\mathrm{P}$ & 13 & 36 & 30 & $10-20$ \\
& $\mathrm{Fe}$ & 192 & 239 & 121 & $10-100$ \\
& $\mathrm{Cu}$ & 28 & 30 & 14 & $2-20$ \\
& $\mathrm{Zn}$ & 8,0 & 7,0 & 6,0 & $2-10$ \\
& $\mathrm{Mn}$ & 52 & 100 & 47 & $5-50$ \\
\hline
\end{tabular}

*T1= Tratamiento en macetas, $\mathrm{T} 2=$ Tratamiento en parcela de campo. Ref= Bertsh (1996) citado por Peña (2016). 
CUADRO 2

Relaciones catiónicas del complejo de cambio del suelo posterior a la mineralizaron los rastrojos de vainica (Phaseolus vulgaris) durante 30 días

\begin{tabular}{|c|c|c|c|c|c|}
\hline \multirow{2}{*}{ Unidad } & \multirow{2}{*}{ Variable } & \multicolumn{3}{|c|}{ Valores } & \multirow{2}{*}{ Ref. } \\
\hline & & Inicial 0 días & T1 20 días & T2 30 días & \\
\hline \multirow{7}{*}{$\mathrm{cmol}(+) / \mathrm{L}$} & $\mathrm{Ca}+\mathrm{Mg}+\mathrm{K}$ & 8,6 & 6,73 & 11,13 & $10-40$ \\
\hline & $\mathrm{Ca} / \mathrm{Mg}$ & 4,3 & 3,8 & 3,72 & $2-5$ \\
\hline & $\mathrm{Ca} / \mathrm{K}$ & 10,83 & 4,93 & 11,23 & $5-25$ \\
\hline & $(\mathrm{Ca}+\mathrm{Mg}) / \mathrm{K}$ & 13,33 & 6,23 & 14,24 & $10-40$ \\
\hline & $\mathrm{Mg} / \mathrm{K}$ & 2,5 & 1,29 & 3,01 & $2,5-15$ \\
\hline & SA & 1,1 & 5,1 & 1,76 & $10-50$ \\
\hline & CICE & 8,7 & 5,87 & 11,33 & - \\
\hline
\end{tabular}

*T1= Tratamiento en macetas, $\mathrm{T} 2=$ Tratamiento en parcela de campo. CICE = capacidad de intercambio catiónico efectiva.

SA: \% saturación de acidez. Ref= Bertsh (1996) citado por Peña (2016).

\section{CUADRO 3}

Contenido de carbono y nitrógeno en el suelo, posterior a la mineralización de los rastrojos de vainica (Phaseolus vulgaris) en dos tratamientos

\begin{tabular}{cccc} 
Tratamiento & \multicolumn{3}{c}{ Variable } \\
Valor inicial & \% & $\% \mathrm{~N}$ & $\mathrm{C} / \mathrm{N}$ \\
$\mathrm{T} 1$ & 3,3 & 0,56 & 5,94 \\
$\mathrm{~T} 2$ & 2,8 & 0,42 & 6,67 \\
\hline
\end{tabular}

${ }^{*} \mathrm{~T} 1=$ Tratamiento en macetas, $\mathrm{T} 2=$ Tratamiento en parcela de campo.

\section{CUADRO 4}

Porcentaje de contenido de nitrógeno, carbono y relación $\mathrm{C} / \mathrm{N}$ de las muestras de los rastrojos de vainica (Phaseolus vulgaris) durante 30 días de mineralización

\begin{tabular}{lcccc} 
& \multicolumn{4}{c}{ Muestreo (días) } \\
\multicolumn{1}{r}{ Muestreo } & 0 & 10 & 20 & 30 \\
Nitrógeno & $4,3 \%$ & $1,27 \%$ & $1,63 \%$ & $0,9 \%$ \\
Carbono & $38,15 \%$ & $14,22 \%$ & $18,29 \%$ & $11,56 \%$ \\
Relación C/N ( \pm D.E.) & $8,86 \pm 0,04$ & $11,23 \pm 0,14$ & $11,20 \pm 0,26$ & $12,90 \pm 0,31$ \\
\hline
\end{tabular}

y nitrógeno disminuyó en ambos tratamientos, presentando inicialmente un valor de 3,3\% de carbono, en T1 de $2,8 \%$ y en $\mathrm{T} 2$ de 3,2 ; por su parte en nitrógeno se presentó inicialmente un valor de $0,56 \%$, disminuyendo en $\mathrm{T} 1$ a $0,42 \%$ y en $\mathrm{T} 2$ a $0,55 \%$.

Mineralización de los rastrojos de vainica: En el Cuadro 4 se muestran los valores en porcentaje de nitrógeno; en el cual se presentó inicialmente un porcentaje de nitrógeno en las hojas de vainica de 4,3\%, disminuyendo progresivamente.

El Cuadro 4 también muestra el contenido de carbono en porcentaje de los rastrojos de vainica que se encontraban en macetas, presentando inicialmente $38,15 \%$, y disminuyendo progresivamente del mismo modo que el nitrógeno comentado anteriormente.

Con respecto a la relación $\mathrm{C} / \mathrm{N}$ en los rastrojos se puede apreciar en el Cuadro 4, que hubo un aumento progresivo, ya que inicialmente se presentó una relación $\mathrm{C} / \mathrm{N}$ de $8,86 \%$, aumentando progresivamente.

En la figura 1 se muestra un análisis del contenido de carbono y nitrógeno en gramos durante los 30 días de mineralización.

El porcentaje de materia seca de los rastrojos se muestra en el Cuadro 5, observándose un comportamiento 


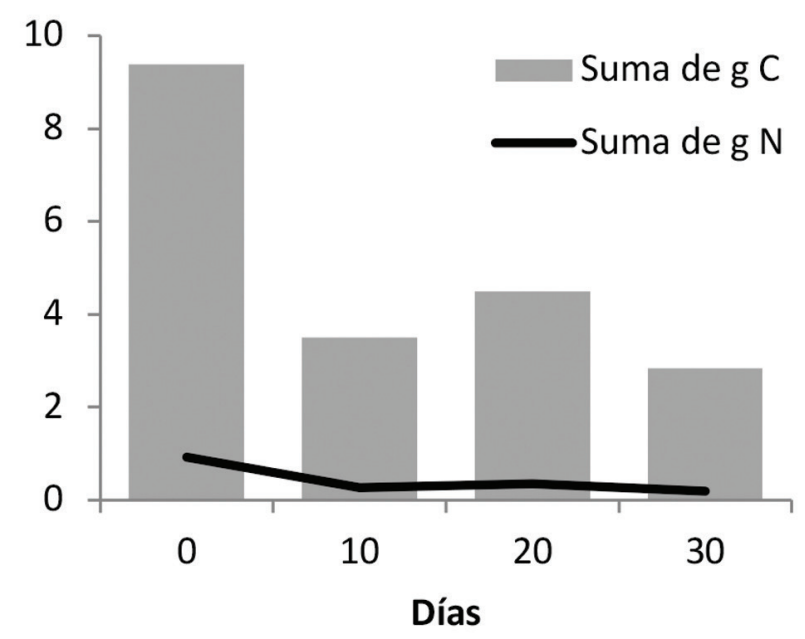

Fig 1. Contenido de nitrógeno y carbono en gramos de las muestras de los rastrojos de vainica (Phaseolus vulgaris) durante 30 días de mineralización.

\section{CUADRO 5}

Materia seca calculada durante la mineralización del rastrojos (Phaseolus vulgaris)

\begin{tabular}{cccc} 
Muestreo & \multicolumn{2}{c}{ Peso $(\mathrm{g})$} & \% materia \\
húmedo & Peso seco & seca \\
Al inicio & 25 & 5 & 20 \\
A los 10 días & 30 & 9 & 30 \\
A los 20 días & 50,1 & 8,5 & 16,9 \\
A los 30 días & 45,2 & 8,0 & 17,7 \\
\hline
\end{tabular}

poco uniforme ya que se presenta un porcentaje de materia seca al inicio de $20 \%$, aumentando a los 10 días a $30 \%$, disminuyendo nuevamente a los 20 días a $16,9 \%$, y aumentando nuevamente a los 30 días con $40,26 \%$.

\section{DISCUSIÓN}

Características químicas del suelo al inicio y al final del experimento: Posterior a la mineralización de los rastrojos de vainica se destaca que en las características químicas del suelo (Cuadro 1) hubo un aumento del potasio $(K)$ y el fosforo $(P)$ en ambos tratamientos, esto motivo del aumento en el nitrógeno del suelo ya que como bien lo indica Fuentes y Gonzáles (2007) se presenta un efecto llamado regulador, mejorando la asimilación del potasio y del ácido fosfórico.

Con respecto al $\mathrm{pH}$ se encontró que el valor de $\mathrm{pH}$ cambio de 5,3 a 5,5 (T1) y 5,6 (T2), encontrándose entre el valor de referencia, Sanclemente (2012) y Monsalve, Gutiérrez, y Cardona (2017) mencionan que en estos rangos se mantiene una buena actividad biológica y por ende una buena mineralización de la materia orgánica. Por su parte la acidez intercambiable, sube dos valores en T2 y un valor en T1, debido a los ácidos orgánicos liberados, los cuales son responsables de la disolución y movimiento del hierro, manganeso y aluminio, ocasionando el proceso de hidrolisis, liberando iones hidronio (Kass, 2007).

En las relaciones catiónicas (Cuadro 2) se puede apreciar que la suma de bases en T2 se encuentra balancea$\mathrm{da}$, pero en $\mathrm{T} 1$ se presenta un desbalance el cual se debe a la disminución en niveles de calcio, del mismo modo sucede con la relación $\mathrm{Ca} / \mathrm{K},(\mathrm{Ca}+\mathrm{Mg}) / \mathrm{K}$ y Mg/K debido a su relación con el aumento de potasio que se presentó posterior a la mineralización de los rastrojos, a pesar de ello la relación $\mathrm{Ca} / \mathrm{Mg}$ se encuentra en balance. Cabe destacar que las relaciones en $\mathrm{T} 2$ se encuentran balanceadas debido a que los niveles de potasio y calcio en este tratamiento si se encuentran entre los valores de referencia.

Además, en el Cuadro 3 se puede observar que el contenido de nitrógeno en el suelo posterior a la mineralización de los rastrojos de vainica es alto, ya que como bien lo indica Andrades (2012) valores de nitrógeno en el suelo mayores a $0,30 \%$ son considerados muy altos y los resultados sobrepasan esta referencia teniendo valores de $0,42 \%$ a $0,56 \%$.

Mineralización de los rastrojos de vainica: El contenido de nitrógeno en los rastrojos de vainica (Phaseolus vulgaris) (Cuadro 4) sobrepasa los valores encontrados por Escalante y Rodríguez (2015) ya que mencionan que el contenido de nitrógeno en la lámina foliar es de 2,1\%, y según los análisis realizados se encontraron valores de $4,3 \%$ inicialmente, el cual fue disminuyendo con respecto al tiempo en el proceso de mineralización.

Además, se puede indicar que a los 30 días se marcó la mayor aportación de nitrógeno teniendo un porcentaje de nitrógeno final en los rastrojos de $0,9 \%$, que inicialmente era de $4,3 \%$, por lo que hubo un aporte de $3,4 \%$ de nitrógeno, que representa el $79,07 \%$ del total de nitrógeno. Del mismo modo se puede interpretar con el nitrógeno en gramos (Fig. 1), en el cual inicialmente se presentaban valores de $0,22 \mathrm{~g}$ quedando finalmente a los 30 días con $0,07 \mathrm{~g}$, teniendo un aporte de $0,15 \mathrm{~g}$ por gramo de materia seca de rastrojo.

Debido a ello se logra establecer que a los 30 días de mineralización de los rastrojos es cuando se presenta la mayor actividad microbiana, y por ende la mejor descomposición de los rastrojos y disponibilidad de nitrógeno, teniendo con ello la mayor aportación de nitrógeno, coincidiendo con las investigaciones realizadas 
por Muñoz (2013) que indica que esta planta posee un alto rendimiento en mineralización.

Por otra parte, el contenido de carbono de los rastrojos (Cuadro 4) disminuyó en mayor cantidad a los 30 días presentando valores de 11,56\%; marcando el mayor aprovechamiento de carbono y concordando con el mayor aporte de nitrógeno, lo que indica la mayor presencia de la actividad microbiana encargada de la descomposición de los rastrojos.

En términos de aprovechamiento se puede indicar que del total de carbono que contenían los rastrojos de vainica, se obtuvo un aprovechamiento de carbono a los 10 días de $63,24 \%$, a los 20 días de $52,05 \%$ y a los 30 días de $69,7 \%$, coincidiendo con Bonadeo, Moreno, Bongiovanni, Marzari, y Ganum (2017) que indican que en los procesos de mineralización aeróbica se aprovecha más que en condiciones anaeróbicas en las cuales los organismos utilizan apenas del 2 al $5 \%$.

Con respecto a la relación carbono nitrógeno (Cuadro 4) se determinó un comportamiento en aumento ya que inicialmente se presentaban valores de $8,86 \%$, teniendo a los 30 días una relación $\mathrm{C} / \mathrm{N}$ de $12,90 \%$, esto debido a la mineralización de los rastrojos de vainica y su aporte de nitrógeno al suelo, teniendo la pérdida progresiva de este nutriente en los rastrojos, cabe mencionar que esta se encuentra entre el rango adecuado ya que según las investigaciones realizadas por Sanclemente (2012) y Portilla (2012) la relación carbono nitrógeno debe encontrase en parámetros $\mathrm{C} / \mathrm{N}$ menores a 25 pues es aquí donde se favorece la mineralización, ya que con valores $\mathrm{C} / \mathrm{N}$ mayores a 30 se presenta la inmovilización de nitrógeno debido a la competencia intensa de los microorganismos por nitrógeno disponible.

Además, se puede concluir, según lo mencionado por Studdert y Echeverría (2006) que los residuos de vainica (Phaseolus vulgaris), al presentar una concentración de nitrógeno importante y poseer una baja relación $\mathrm{C} / \mathrm{N}$, son fácilmente descompuestos y se aporta gran cantidad de nutrientes durante los primeros ciclos del cultivo siguiente.

Por su parte las hojas de los rastrojos de vainica (Phaseoulus vulgaris) a los 0 días (Cuadro 5) contenían un $20 \%$ de materia seca, mostrando una disminución al final del experimento ya que contenían solamente un $17,7 \%$, este comportamiento se puede atribuir a la mineralización de los rastrojos y la perdida de humedad, así como su descomposición.

Asimismo, tomando como referencia el porcentaje de materia seca en los rastrojos se puede apreciar en la figura 1 que hay una reducción progresiva a través del tiempo de nitrógeno y carbono, esto motivo de la mineralización y su aporte de nitrógeno al suelo, así como las necesidades de carbono que presentan los microorganismos para realizar con eficacia este proceso.

En el presente trabajo se concluye que a los 30 días se marcó la mayor aportación de nitrógeno y aprovechamiento de carbono, así como la mayor actividad microbiana, teniendo un aporte de nitrógeno final del 79,07\% del total de nitrógeno que contenían los rastrojos, por tal motivo se indica que los rastrojos de vainica funcionan como abono verde, ya que presentan concentraciones importantes de nitrógeno y una relación $\mathrm{C} / \mathrm{N}$ baja favoreciendo el proceso de mineralización.

Además, se recomienda el uso de la vainica (Phaseolus vulgaris) para ser utilizada como abono verde, pues posee una buena mineralización y aporte de nutrientes para el mejoramiento de la fertilidad del suelo; dicha incorporación debe realizarse considerando que la mayor disponibilidad de nutrientes se da a los 30 días de mineralización, con el fin de planear la siembra según los requerimientos del cultivo. Cabe destacar que se deben de tener en cuenta los factores climatológicos ya que influyen sobre la mineralización retardando o favoreciendo el proceso, así como los factores sanitarios mencionando entre ellos las enfermedades y plagas presentes en el cultivo ya que pueden limitar la eficacia del uso de los rastrojos como abono verde.

\section{AGRADECIMIENTOS}

Agradecemos a la Universidad Estatal a Distancia (UNED), por haberme dado los recursos para lograr concluir mi carrera universitaria. A mi supervisora la Msc. Paola Brenes Rojas, al Dr. Wagner Peña por su asesoría, al Instituto Nacional de Tecnología Agropecuaria (INTA), por su colaboración en la elaboración y financiamiento de los análisis de laboratorio, al Instituto Meteorológico Nacional por suministrar los datos climáticos, a mi familia, amigos y compañeros por su apoyo incondicional.

\section{REFERENCIAS}

Andrades, M. (2012). Prácticas de edafología y climatología. Recuperado de https://dialnet.unirioja.es/descarga/libro/194611.pdf

Bonadeo, E., Moreno, I., Bongiovanni, M., Marzari, R., \& Ganum, M. (2017). El sistema suelo planta. Recuperado de https://www.unrc.edu.ar/unrc/comunicacion/editorial/ repositorio/978-987-688-204-0.pdf

Delgado, R., Salvador, J., Morales, E., López, J., \& Rocandio, M. (2015). Producción y rentabilidad del frijol ejotero 
(Phaseolus vulgaris L.) asociado a maíz en función de la densidad y el nitrógeno en clima templado. Revista de la Facultad de Ciencias Agrarias, 47(2), 15-25. Recuperado de http://www.redalyc.org/articulo.oa?id=382842590002

Escalante, A., \& Rodríguez, T. (2015). Rendimiento, eficiencia agronómica, extracción de nitrógeno en Phaseolus vulgaris (I.) y prácticas agrícolas (tesis de postgrado). Colegio de postgraduados, México.

Figueroa, A., Álvarez, J., Forero, A., Salamanca, C., \& Pinzón, L. (2012). Determinación del nitrógeno potencialmente mineralizable y la tasa de mineralización de nitrógeno en materiales orgánicos. Temas Agrarios, 17(1), 32-43. DOI: 10.21897/rta.v17i1.694

Fuentes, W., \& Gonzales, O. (2007). Estimación de la mineralización neta de nitrógeno del suelo en sistemas agroforestales y a pleno sol en el cultivo del café (coffea arabica l.), en el pacífico de Nicaragua, departamento de Carazo (trabajo de diploma). Universidad Nacional Agraria, Managua, Nicaragua.

Guanche, A. (2012). Los abonos verdes. Recuperado de http://www.agrocabildo.org/publica/Publicaciones/ agec_454_abonos_verdes.pdf

INEC (Instituto Nacional de Estadística y Censos). (2014). VI Censo Nacional Agropecuario. Recuperado de http:// www.mag.go.cr/bibliotecavirtual/U40-10581.pdf

Jaramillo, C. (2016). Mineralización de la gallinaza y de los restos de cosecha en el suelo: Aplicación al cultivo de la coliflor en la Huerta de Valencia (tesis doctoral). Universidad Politécnica de Valencia, España.

Kass, D. (1996). Fertilidad de suelos y fertilizantes. San José, Costa Rica: EUNED.

MAG (Ministerio de Agricultura y Ganadería). (1999). Vainica (Phaseoulus vulgaris). Recuperado de http://www.mag. go.cr/bibliotecavirtual/F01-0658vainica.pdf

Martínez, J., Galantini, J., López, F., \& Duval, M. (2014). Índices de mineralización del nitrógeno. Boletín del Cerdos,
25, 15-25. Recuperado de https://ri.conicet.gov.ar/ handle/11336/11578

Monsalve, O., Gutiérrez, J., \& Cardona, W. (2017). Factores que intervienen en el proceso de mineralización de nitrógeno cuando son aplicadas enmiendas orgánicas al suelo. Revista Colombiana de Ciencias Hortícolas, 11 (1), 200209. DOI: 10.17584/rcch.2017v11i1.5663

Muñoz, R. (2013). Leguminosas como alternativa para la producción de maíz en el Ejido La Bella Ilusión, Maravilla Tenejapa, Chiapas (tesis de maestría). El Colegio de la Frontera Sur, México.

PIMA (Programa Integral de Mercadeo Agropecuario). (2016). Análisis del consumo de frutas, hortalizas, pescado y mariscos en los hogares costarricenses. Recuperado de http://www.pima.go.cr/wp-content/uploads/2017/07/ Analisis-Consumo.pdf

Peña, W. (2016). Edafología del trópico. San José, Costa Rica: EUNED.

Piccinetti, C., García, S., \& Perticari, A. (2015). Estimación del aporte de nitrógeno biológico en el cultivo de poroto en tres localidades del Noa. Recuperado de https://inta.gob. ar/sites/default/files/inta-piccinetti-ii.pdf

Portilla, A. (2012). Evaluación de la biodisponibilidad del nitrógeno en cultivo de fréjol arbustivo (Phaseolus vulgaris l.) con cobertura y bajo sistemas de labranza de conservación, en la microcuenca del río Alumbre - Bolívar (tesis de grado). Universidad Central de Ecuador.

Sanclemente, O. (2012). Materia orgánica del suelo. Recuperado de https://slideplayer.es/slide/3172712/

Studdert, G ., \& Echeverría, H. (2006). Relación entre el cultivo antecesor y la disponibilidad de nitrógeno para el trigo en la rotación.CISuelo, 24(1), 1-8. Recuperado de https://www. researchgate.net/publication/242132524_Relacion_entre_el_cultivo_antecesor_y_la_disponibilidad_de_nitrogeno_para_el_trigo_en_la_rotacion

Vargas, G. (2011). Botánica general: de los musgos hasta los árboles. San José, Costa Rica: EUNED. 